2. A. Malcev, On the immersion of an algebraic ring into a field, Math. Ann. vol. 113 (1936-1937) pp. 686-691.

3. J. S. Shepherdson, Inverses and zero divisors in matrix rings, Proc. London Math. Soc. (3) vol. 1 (1951) pp. 71-85.

UNIVERSITY OF NEBRASKA

\title{
A SPLITTING THEOREM AND THE PRINCIPAL IDEAL THEOREM FOR SOME INFINITELY GENERATED GROUPS
}

\section{EUGENE SCHENKMAN ${ }^{1}$}

The first theorem of this paper is an extension of a splitting theorem for finite groups (cf. [6]) to include periodic groups certain of whose Sylow subgroups contain no elements of infinite height.

The second theorem is an extension of the principal ideal theorem for finitely generated groups (cf. [7]) to include all groups except again that some of the Sylow subgroups contain no elements of infinite height. A counter example will show that this latter restriction is necessary for both theorems.

In the first draft of the paper the second theorem was stated for periodic groups, the proof being based on the first theorem. The present more general statement and proof independent of Theorem 1 are due to the referee to whom I am also indebted for a simplification in the proof of the lemma below.

The Splitting Theorem. As usual $G$ will stand for the group, $G^{\prime}$ for its commutator subgroup, and $G^{*}$ for the intersection of the members $G^{m}$ of the descending series of $G$. $E$ will be the subgroup consisting only of the identity $e$. We let $[a, b]$ stand for $a b a^{-1} b^{-1}$ and refer the reader to [6] for some of the standard identities on commutators that will be needed. In particular we shall need that $[x, h g]=[x, h][x, g]$ if $h$ and $g$ are in an Abelian normal subgroup; and $[x, h z]=[x, h]$ if $[x, z]=e$.

Lemma. Let $G$ be a p-group with no elements of infinite height; let $G / G^{*}$ be finite and let $G^{*}$ be Abelian. Then $G^{*}$ consists only of the identity and $G$ is finite.

Presented to the Society, September 2, 1955; received by the editors May 31, 1955.

${ }^{1}$ I wish to thank Professors Beaumont, Curtis, Reiner, and Swift for interesting discussions during the preparation of this paper. Work was supported by the Office of Ordnance Research United States Army Contract No. DA-36-034-ORD-1622RD. 
Proof. Let $b \neq e$ be in $G^{*}$; then a suitable power of $b$ has order $p$ and by $\left[3\right.$, p. 180] $G^{*}$ is the direct product of $U$ and a nontrivial finite cyclic group. The index of $U$ in $G$ has $p$-power order and hence so also has the intersection $N$ of the finite number of conjugates of $U$. Thus $N$ is normal in $G$, and $G / N$ is a finite $p$-group. It follows that $(G / N)^{*}$ is the identity, whence $G^{*}$ is contained in $N$ which is a contradiction of the fact that $N$ is contained in $U$, a proper subgroup of $G^{*}$. We must conclude that $G^{*}=E$ and hence $G$ is finite.

We use this lemma to prove the splitting theorem.

THEOREM. Let $G$ be a periodic group such that $G / G^{*}$ has finite order $n$ and let $G^{*}$ be Abelian; suppose also that there are no elements of infinite height in the Sylow subgroups of $G^{*}$ corresponding to the prime divisors of $n$. Then there is a finite nilpotent subgroup $H$ of $G$ such that $H$ intersects $G^{*}$ only in the identity and such that $H$ and $G^{*}$ generate $G$.

Proof. We divide the proof into the following parts. (1) By [4, p. 190], $G$ is locally finite and is generated by $G^{*}$ and finite $p_{i}$-groups $P_{i}$ for $i=1, \cdots, k$.

For any set of representatives of $G / G^{*}$ generates a finite group whose generating Sylow $p$ subgroups we may designate by $P_{1}, \cdots$, $P_{k}$.

(2) Since $G$ is locally finite and solvable, by [5] the weakly central elements of $G$ comprise a normal subgroup $N$ of $G$ (cf. also [2]), $N$ containing $G^{*}$ such that $N$ is a direct product of $p$ groups $N_{p}$.

We define here an element $a$ of $G$ to be weakly central (cf. also [1]) if for any $g$ in $G[\cdots[g, a] \cdots a]$ (where there are $n a^{\prime}$ s) $=e$ for some $n$ depending on $g$. This definition is slightly more inclusive than that given in [5], but the theorems and proofs then follow verbatim with this definition.

(3) If $P$ is any $p$-subgroup of $G$ then $P^{*}=E$. For let $P$ be imbedded in a Sylow $p$-group $S_{p}$ of $G$. Then $S_{p}$ is Abelian except for the primes $p_{i}$ of (1). Such a Sylow $p_{i}$-subgroup $S_{p_{i}}$, however is generated by a finite group $P_{i}$ such as in (1) and $S_{p_{i}} \cap G^{*}$. Let $\bar{P}_{i}$ be the group generated by $P_{i}$ and $S_{p_{i}}^{*}$. Then by the second identity for commutators at the beginning of this section we see that $\bar{P}_{i}^{*}=S_{p_{i}}^{*}$. Thus $\bar{P}_{i}^{*}$ has finite index in $\bar{P}_{i}$. It follows from the lemma that $\bar{P}_{i}^{*}=E$. Hence also $P^{*}$ is $E$ since $P^{*} \subseteq S_{p_{i}}^{*}=\bar{P}_{i}^{*}=E$.

(4) If $K$ is generated by $N$ and an element $x$ of prime power order $q^{k}, x$ not in $N$, then $K^{*} \neq E$, and for all primes $p$ not $q$ if $M_{p}$ is the group generated by $N_{p}$ and $x$ then $M_{p}^{*}=N_{p} \cap K^{*}$.

For $x$ is not weakly central by (2) and hence for some $g$ in $N, x$ and $g$ generate a non-nilpotent finite group so that $K^{*}$ can clearly not be 
$E$. For the second assertion we note that $N_{p}$ is normal in $K$ and hence $\left[N_{p} \cap K^{*}, K\right]=\left[N_{p} \cap K^{*}, M_{p}\right]$ by the second identity listed above for commutators.

(5) If $M_{p}$ is as in (4) and if also $M_{p}^{*}$ has finite index in $M_{p}$ then $M_{p}^{*}=R_{p}^{*}$ where $R_{p}$ is the group generated by $M_{p}^{*}$ and $x$.

Clearly $M_{p}^{*} \supset R_{p}^{*}$. We must show that $M_{p}^{*} \subset R_{p}^{*}$. Since $M_{p}^{*}$ has finite index, $M_{p} / M_{p}^{*}$ is nilpotent and is a direct product of a $p$-group by a $q$-group. It follows that $x M_{p}{ }^{*}$ is normal in $M_{p} / M_{p}{ }^{*}$ and therefore $R_{p}$ and consequently also $R_{p}^{*}$ are normal in $M_{p}$. Thus modulo $R_{p}^{*}$, $x$ is weakly central so that $M_{p} / R_{p}^{*}$ is generated by weakly central elements and therefore by (2) is the direct product of p-groups. It follows that $\left(M_{p} / R_{p}^{*}\right)^{*}=E$ or $M_{p}^{*} \subset R_{p}^{*}$ as was asserted.

(6) If $x, N_{p}$, and $M_{p}$ are as in (4) and if $Z_{p}$ is the intersection of the centralizer of $x$ with $N_{p}$ then $M_{p}^{*}$ and $Z_{p}$ generate $N_{p}$ and $M_{p}^{*} \cap Z_{p}$ $=E$.

For let $y$ be in $N_{p}$. Then $y$ and $x$ generate a finite group which modulo $M_{p}^{*}$ is nilpotent and hence a direct product of $p$-groups; consequently $[y, x]$ is in $M_{p}^{*}$. But $M_{p}^{*}=R_{p}^{*}$ so that by the lemma of [6] $[y, x]=[t, x]$ for some $t \in M_{p}^{*}$. Then $y t^{-1} \in Z_{p}$ since $\left[y t^{-1}, x\right]$ $=[y, x]\left[t^{-1}, x\right]=[y, x][t, x]^{-1}=e$. Hence $y$ is in $\left\{M_{p}^{*}, Z_{p}\right\}$ as asserted.

To show that $M_{p}^{*} \cap Z_{p}=E$ we assume that $z \neq e$ is in $M_{p}^{*} \cap Z_{p}$. Then $z=[t, x]$ as before with $t$ in $M_{p}^{*}$ but since $z$ is in $Z_{p},[t, x]$ commutes with $x$; and since $z$ is in the Abelian normal group $M_{p}^{*},[t, x]$ commutes with $t$. It follows that $[t, x]$ is in the center of the group generated by $t$ and $x$ which is consequently a nilpotent group. Then $[t, x]=e$ since $t$ and $x$ have relatively prime orders in this nilpotent group. Thus $z$ is $e$ as was to be shown.

(7) It follows from (6) that if $G$ is generated by $N$ and an element $x$ of $q$ power order then $G$ is a split extension of $G^{*}$ by the nilpotent group generated by the Sylow $q$-subgroup $S_{q}$ and all the $Z_{p}$ defined as in (6) for all primes $p$.

(8) We proceed by induction on the order of $G / N$. Let $K$ be normal in $G$ so that $K$ is generated by $N$ and an element $x$ of $q$ power order. Then $K^{*}$ is a nontrivial normal subgroup of $G$ and the induction hypothesis applies to $G / K^{*}$. Thus $G / K^{*}=T / K^{*} \cdot G^{*} / K^{*}$ with $T \cap G^{*}$ $\subset K^{*}$ so that $T^{*}=K^{*}$. Then $T$ contains a finite group $V$ which with $T^{*}$ generates $T$; by the splitting theorem of [6], $V$ contains a nilpotent group $H$ so that $H$ and $T^{*}$ generate $T$.

We will now show that $H \cap T^{*}=E$. For if $h$ were in $H$ as well as in $T^{*}$ then $h$ would have order prime to $q$ since $T^{*}=K^{*}$ and $x$ has $q$ power order. But $H$ would have an element $x^{\prime}$ of $q$ power order $=x g^{*}$ 
where $g^{*}$ is in $G^{*}$ so that $[h, x]=\left[h, x^{\prime}\right]$. But this is impossible for $\left[h, x^{\prime}\right]$ should be $e$ since $h$ and $x^{\prime}$ have relatively prime orders in the nilpotent group $H$, on the other hand $[h, x] \neq e$ since $h$ is in $K^{*}$.

This allows us to finish the proof. For $H$ and $G^{*}$ generate $G$ since $H$ and $T^{*}$ generate $T$ and $T$ and $G^{*}$ generate $G$. Also $H \cap G^{*}=E$ since $\left(H \cap G^{*}\right) \subset\left(T \cap G^{*}\right) \subset K^{*}$ and $H \cap K^{*}=E$.

\section{The Principal Ideal Theorem.}

Theorem. Let $G$ be a group so that $G / G^{\prime}$ has finite order $n$ and so that the derived group $G^{\prime \prime}=E$. Suppose also that there are no elements of infinite height in the Sylow subgroups of $G^{\prime}$ corresponding to the prime divisors of $n$. Then the transfer of $G$ into $G^{\prime}$ is $E$ (cf. [7, Chapter V]).

Proof. For suppose to the contrary that there is an element $x$ in $G$ whose transfer $y$ is an element of order $p, p$ a prime divisor of $n$. Then $G^{\prime}$ can be decomposed into a direct sum of $A$ and $B$ where $A$ is a finite group containing $y$ (cf. [3, p. 205]). Then $B$ has finite index in $G$ and hence the intersection $N$ of the finite number of conjugates of $B$ is a normal subgroup with finite index in $G$. But $y$ is not in $N$ and we have then that the transfer of $G / N$ is not the identity contradicting the Principal Ideal Theorem of [7] since $G / N$ is finite.

Counter example. The following group similar to that in $[7, \mathrm{p} .147]$ serves to show that the assumption about elements of infinite height is necessary in both the above theorems and also in the lemma.

Let $U$ be the group of roots of unity $u$ whose orders are powers of 2 (i.e. $U$ is a $(2, \infty)$ group), and let $G=\{U, j\}$ be the extension over $U$ of index 2 defined by $j^{2}=-1$ and $j u j^{-1}=u^{-1}$. Then it is easy to verify that $G^{\prime}=G^{*}=U$ and that $G^{\prime \prime}=(1)$ and that none of our results can apply to this group.

\section{BiBLIOGRAPHY}

1. K. W. Gruenberg, Two theorems on Engel groups, Proc. Cambridge Philos. Soc. vol. 49 (1953) pp. 377-380.

2. K. A. Hirsch, Ueber lokal-nilpotente Gruppen, Math. Zeit. vol. 63 (1955) pp. 290-294.

3. A. G. Kurosch, The theory of groups, vol. 1, trans. by K. A. Hirsch, New York, Chelsea, 1955.

4. - Theory of groups, vol. II, New York, 1956.

5. E. Schenkman, $A$ generalization of the central elements of a group, Pacific Journal of Mathematics vol. 3 (1953) pp. 501-504.

6. - The splitting of certain solvable groups, Proc. Amer. Math. Soc. vol. 6 (1955) pp. 286-290. 1949.

7. H. Zassenhaus, Theory of groups, trans. by S. Kravetz, New York, Chelsea,

Louisiana State University and Agricultural and Mechanical College 